\title{
High expression of DII4 and CD44V6 is associated with clinicopathological characteristics and poor prognosis in osteosarcoma patients
}

\author{
Haiyang Xie ${ }^{1}$, Ye Wang ${ }^{1}$, Ting Yu $^{2}$, Cheng Jiang ${ }^{1}$, Peng Wei ${ }^{1}$, Yingbo Zhang ${ }^{1}$ \\ ${ }^{1}$ Department of Orthopedics, Affiliated Hospital of North Sichuan Medical College, Nanchong, China; ${ }^{2}$ Department of Infection, Affiliated Hospital \\ of North Sichuan Medical College, Nanchong, China \\ Contributions: (I) Conception and design: H Xie, Y Zhang; (II) Administrative support: Y Wang, T Yu; (III) Provision of study materials or patients: H \\ Xie, Y Wang, T Yu, C Jiang, P Wei; (IV) Collection and assembly of data: All authors; (V) Data analysis and interpretation: H Xie, C Jiang, P Wei, Y \\ Zhang; (VI) Manuscript writing: All authors; (VII) Final approval of manuscript: All authors. \\ Correspondence to: Yingbo Zhang. Department of Orthopedics, Affiliated Hospital of North Sichuan Medical College, 63 Cultural Road, Shunqing \\ District, Nanchong, China. Email: zhangyingbo333@163.com.
}

Background: Osteosarcoma is a malignant tumor with a poor prognosis. Dll4 and CD44V6 are involved in the regulation of tumor development. This study aims to analyze the expression of D114 and CD44V6 in osteosarcoma and the correlation with clinicopathological characteristics and prognosis.

Methods: Clinical data of 47 patients with osteosarcoma admitted to our hospital from April 2016 to May 2018 and confirmed by surgery or pathological examination were retrospectively analyzed. Osteochondroma and normal bone tissues from these cases were taken as controls. The protein levels of D114 and CD44V were detected by immunohistochemistry (IHC). The relationship between the expression of D114 and CD44V and the clinicopathological characteristics of patients with osteosarcoma was analyzed. An unconditional multifactor logistic regression model was used to analyze the risk factors affecting the prognosis of patients with osteosarcoma.

Results: The positive expression rates of Dll4 and CD44V6 in osteosarcoma were 85.11\% (D114) and 91.49\% (CD44V6), which were significantly higher than those in normal bone tissues of $18.52 \%$ (D114) and $7.41 \%$ (CD44V6), and in osteochondroma of 22.22\% (D114) and 7.41\% (CD44V6). The expression of CD44V6 and Dll4 were related to the Enneking stage and metastasis $(\mathrm{P}<0.05)$, and the expression of Dll4 was closely related to the degree of tumor differentiation $(\mathrm{P}<0.05)$. The 2-year survival rates of patients with D114 or CD44V6 positive expression were $25.00 \%$ and $21.88 \%$, respectively, which were significantly lower than those with D114 or CD44V6 negative expression $(85.71 \%$ and $93.33 \%)(\mathrm{P}<0.05)$. The results of the unconditional multivariate logistic regression analysis showed that low tumor differentiation, high Enneking stage, metastasis, and positive expression of Dll4 and CD44V6 were risk factors affecting the mortality rate and prognosis of patients with osteosarcoma $(\mathrm{P}<0.05)$.

Conclusions: The positive expression rate of Dll4 and CD44V6 in osteosarcoma is high, and the expression level is closely related to the degree of tumor differentiation, Enneking stage, and metastasis. D114 and CD44V6 are risk factors affecting the survival prognosis for patients. Therefore, in clinical settings, the prognosis for patients could be evaluated by detecting the expression of D114 and CD44V6.

Keywords: Osteosarcoma; Dll4 protein; CD44V6; clinicopathological characteristics; prognosis

Submitted Dec 10, 2020. Accepted for publication Feb 03, 2021.

doi: $10.21037 /$ tcr-21-31

View this article at: http://dx.doi.org/10.21037/tcr-21-31 


\section{Introduction}

As a malignant tumor, osteosarcoma originates in mesenchymal tissue and is characterized by the production of spindle-shaped stromal cells of bone-like tissue, which can be divided into primary and secondary osteosarcomas (1). Osteosarcoma is common in young people aged 10 to 20 years old, and mostly appears in the long tubular metaphyseal end, distal femur, tibia and proximal humerus. The prognosis of osteosarcoma is poor because it is highly malignant, aggressive, prone to lung metastasis in the early stage, and has a high recurrence rate after surgical treatment. Therefore, it is of great importance to study the invasion and metastasis of osteosarcoma $(2,3)$. However, there are few studies on the correlation between the expression of DLL4 protein and CD44v6 and the prognosis of patients with osteosarcoma. CD44 is a type of adhesion molecule that has not yet been categorized, while CD44V6 is a subtype of the CD44 molecule containing a variant exon V6 coding sequence. The abnormal expression of CD44V6 is reported to be closely related to the occurrence and development of osteosarcoma (4). The Dll4 protein is a ligand of Notch receptors and has been reported to be involved in the regulation of tumor angiogenesis and growth (5). Therefore, this retrospective study analyzed the expression of Dll4 and CD44V6 proteins in osteosarcoma and the correlation with clinicopathological characteristics and prognosis. We present the following article in accordance with the REMARK reporting checklist (available at http://dx.doi.org/10.21037/tcr-21-31).

\section{Methods}

\section{General information}

Clinical data from 47 osteosarcoma patients admitted to our hospital from April 2016 to May 2018 were analyzed retrospectively, with all cases confirmed by surgery or pathological examination. Inclusion criteria were as follows: (I) no chemotherapy, radiotherapy or anti-angiogenesis drugs had been used prior to surgery; (II) the clinical data records were complete; and (III) no history of drug allergies. Patients were excluded based on the following criteria: (I) patients with poor blood coagulation function; (II) patients with abnormal heart, liver, kidney or other functions; (III) patients who were unconscious; (IV) patients with immune dysfunction; (V) pregnant women during lactation or pregnancy.

Among the 47 patients included in the study, 27 cases were male, and 20 cases were female. Patients were aged from 8 to 61 years, with an average age of $24.5( \pm 13.05)$ years. According to the Enneking surgical staging system, 13 cases were in stage I, 19 cases were in stage II, and 15 cases were in stage III. The degree of differentiation amongst cases was as follows: 10 cases had high differentiation, 13 cases had moderate differentiation, and 24 cases had low differentiation. In regard to pathological type, 28 cases had osteogenic osteosarcoma, 13 cases had chondroid osteosarcoma and 6 cases had fibroblastic osteosarcoma. Osteochondroma and normal bone tissues from each of the 27 cases were taken as controls. The study was conducted in accordance with the Declaration of Helsinki (as revised in 2013). The study was approved by institutional committee of Affiliated Hospital of North Sichuan Medical College (2019ER(R)108-1) and informed consent was taken from all the patients.

\section{S-P immunobistochemical staining}

S-P immunohistochemical staining was used. Firstly, specimens were prepared by dewaxing and hydrating the tissue sections. Then the expression of Dll4 and CD44V6 proteins was detected according to the S-P immunohistochemical staining methods. The main reagents were mouse anti-human CD44V6 monoclonal antibody (Santa Cruz, USA) and rabbit anti-human Dll4 polyclonal antibody (Abcam, UK). The testing process was strictly in accordance with the manufacturer's instructions. The negative control was the use of PBS (phosphate buffered saline) to replace the primary antibodies.

The protocol for judging the staining result was as follows: the positive expression of D114 and CD44V6 proteins was located in tumor cells whose cytoplasm or cell membrane was brownish yellow, and percentages of positive cells were scored as 0 points for no positive cells, 1 point for positive cells $<10 \%, 2$ points for positive cells between $10-50 \%, 3$ points for positive cells between $50-75 \%$, and 4 points for positive cells $>75 \%$. The degree of staining was scored as 0 points for colorless, 1 point for light yellow staining, 2 points for brown staining and 3 points for tan staining. The total scores were defined as the product of the above 2 types of scores, and positive expression was classified as a total score $>4$ points.

\section{Observation indicators}

In order to observe the expression of D114 and CD44V6 


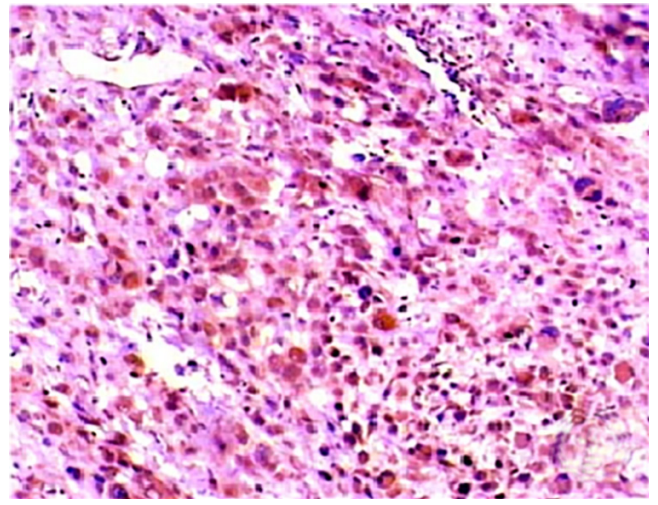

Figure 1 Expression of CD44V6 in osteosarcoma tissues localized in the cell membrane were stained by S-P immunohistochemical staining, and the images were magnified by 400 folds.

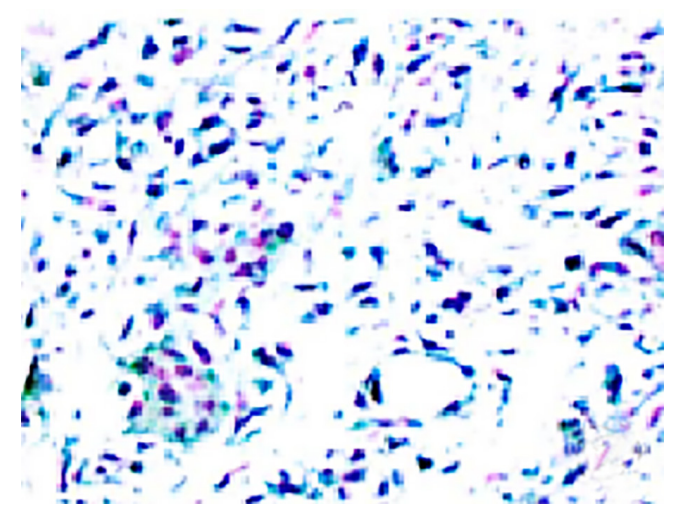

Figure 2 Expression of Dll4 protein in osteosarcoma tissues localized in the cytoplasm were stained by S-P immunohistochemical staining. The images were magnified by 400 folds.

proteins in various bone tissues and analyze their relationship with the clinicopathological characteristics of osteosarcoma, patients were followed up for two years. By analyzing the 2-year survival rate after surgery and the expression level of the D114 and CD44V6 proteins, we could further analyze the relationship between D114 and CD44V6 expression and the risk factors affecting prognosis.

\section{Statistical methods}

The data were statistically analyzed using SPSS 18.0 software (IBM, USA). The measurement data were described as the mean \pm standard deviation $\left(\bar{x}_{ \pm s}\right)$ and analyzed using $t$-tests. The pass rate or composition ratio of the count data was analyzed by the $\chi^{2}$ test. An unconditional multi-factor logistic regression was used to analyze the risk factors affecting the prognosis of osteosarcoma patients. Results with a $\mathrm{P}$ value $<0.05$ were considered to be statistically significant.

\section{Results}

\section{The expression of Dll4 and CD44V6 proteins in various bone tissues}

The positive expression of Dll4 and CD44V6 proteins was located in tumor cell cytoplasm or cell membranes that were brownish yellow (Figures 1,2). The positive expression rates of Dll4 and CD44V6 in osteosarcoma were $85.11 \%$ and $91.49 \%$, respectively, compared with $18.52 \%$ (Dll4) and $7.41 \%$ (CD44V6) in normal bone tissues, and 22.22\% (Dll4) and $7.41 \%$ (CD44V6) in osteochondroma tissues, indicating that the positive expression rates of D114 and CD44V6 in osteosarcoma were significantly higher than those in normal bone tissues and osteochondroma tissues $(\mathrm{P}<0.05$, Table 1).

\section{The relationship between the expression levels of Dll4, CD44V6 and the clinicopathological characteristics of osteosarcoma}

The results showed that the expression of CD44V6 was closely related to the Enneking stage and metastasis $(\mathrm{P}<0.05)$ but did not show a significant relationship with gender, age, tumor diameter, degree of differentiation, or pathological type of osteosarcoma $(\mathrm{P}>0.05)$. Similarly, the expression of Dll4 was significantly related to the degree of tumor differentiation, Enneking stage, and metastasis $(\mathrm{P}<0.05)$ but was not related to gender, age, tumor diameter, or pathological type of osteosarcoma $(\mathrm{P}>0.05$, Table 2$)$.

\section{The relationship between Dll4 and CD44V6 expression and postoperative survival time}

The patients were followed up for two years after surgery, and in that time 22 cases died, resulting in an overall survival rate of $53.19 \%$. The 2 -year survival rates of patients with Dll4 or CD44V6 positive expression were $25.00 \%$ and $21.88 \%$, respectively. The 2 -year survival rates of patients with Dll4 or CD44V6 negative expression were $85.71 \%$ and $93.33 \%$, respectively, which were significantly higher than those with a Dl14 or CD44V6 positive expression $(\mathrm{P}<0.05$, Table 3). Results of the survival analysis showed that patients 
Table 1 Expression of Dll4 and CD44V6 proteins in various bone tissues [n (\%)]

\begin{tabular}{|c|c|c|c|c|c|}
\hline Expression of protein & Normal bone tissue $(n=27)$ & Osteochondroma $(n=27)$ & Osteosarcoma $(n=47)$ & $\chi^{2}$ & $\mathrm{P}$ \\
\hline Negative (-) & 25 (92.59) & 25 (92.59) & $15(31.91)$ & & \\
\hline Positive (+) & $2(7.41)$ & $2(7.41)$ & $32(68.09)^{\#}$ & & \\
\hline DII4 & & & & 42.201 & 0.001 \\
\hline Positive (+) & $5(18.52)$ & $6(22.22)$ & $40(85.11)^{\#}$ & & \\
\hline
\end{tabular}

\#, $\mathrm{P}<0.05$, compared with normal bone tissue or osteochondroma.

Table 2 Relationship between the expression levels of Dll4 and CD44V6 and the clinicopathological features of osteosarcoma [n (\%)]

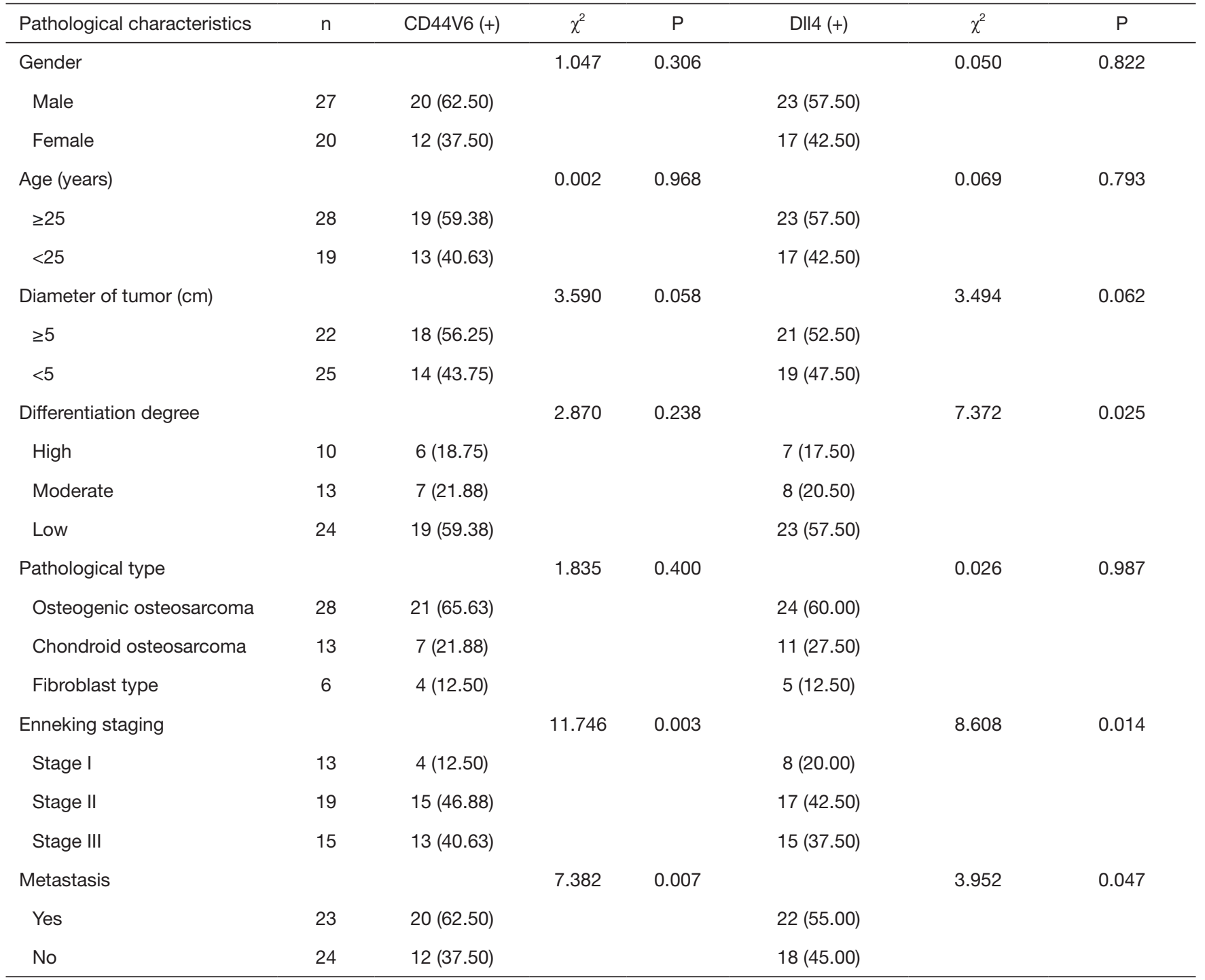


Table 3 Relationship between D114 and CD44V6 expression and postoperative survival time

\begin{tabular}{|c|c|c|c|c|}
\hline Death within 2 years & \multicolumn{2}{|c|}{ CD44V6 } & \multicolumn{2}{|c|}{ DIl4 } \\
\hline Yes & 1 & 25 & 1 & 30 \\
\hline No & 14 & 7 & 6 & 10 \\
\hline Survival rate (\%) & 93.33 & 21.88 & 85.71 & 25.00 \\
\hline
\end{tabular}

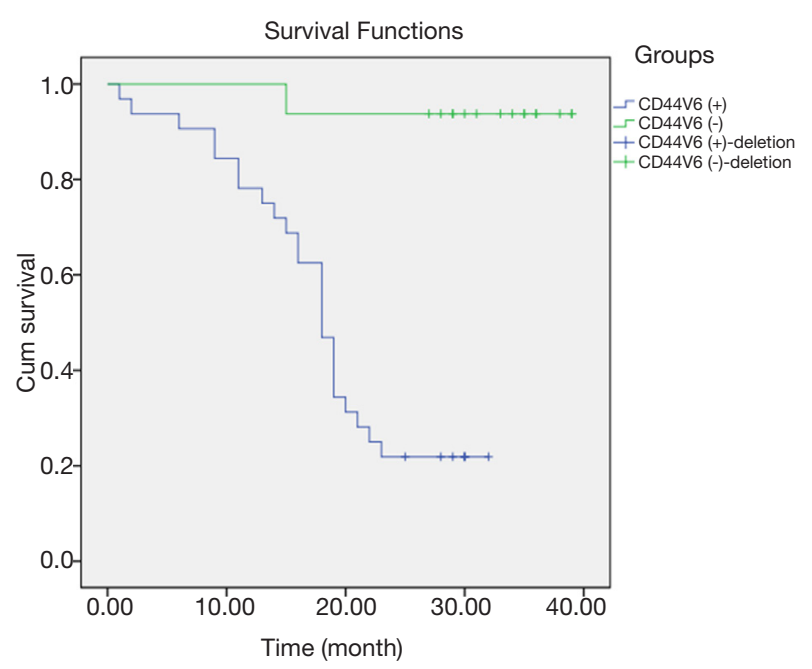

Figure 3 Survival rate analysis of the positive and negative expression of CD44V6.

with Dl14 and CD44V6 negative expressions had a longer survival period, and the Kaplan-Meier survival curves were significantly different from those of patients with Dll4 and CD44V6 positive expressions (Figures 3,4, $\mathrm{P}<0.05$ ).

\section{The analysis of risk factors affecting the prognosis of osteosarcoma patients}

The results showed that tumors with a low differentiation, a high Enneking stage, metastasis, and a positive expression of Dll4 and CD44V6 were risk factors that affected the prognosis of osteosarcoma patients $(\mathrm{P}<0.05$, Table 4$)$.

\section{Discussion}

CD44 is a very widely distributed cell surface transmembrane glycoprotein that can bind to ligands such as hyaluronic acid, mediating the adhesion of cells to cells, and cells to matrix and which displays a broad range of biological functions $(6,7)$. In recent years, a large

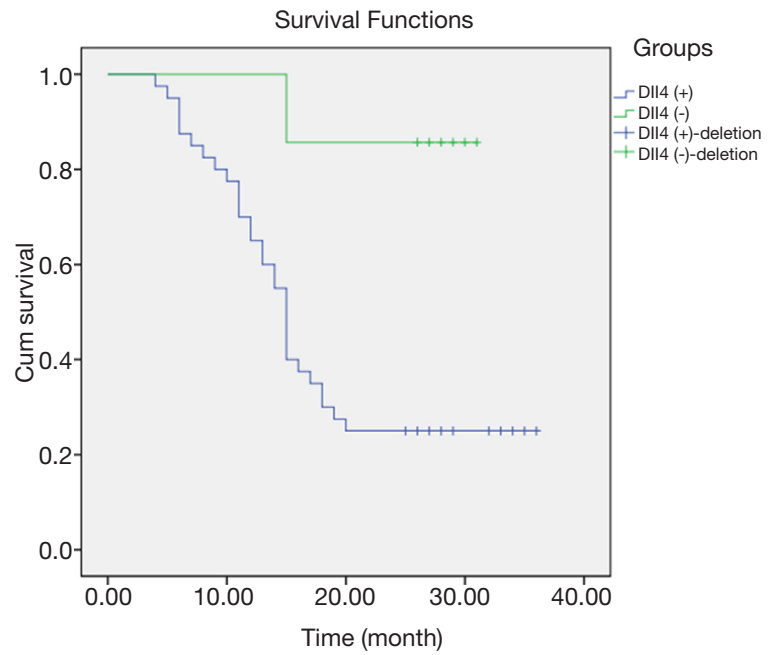

Figure 4 Survival rate analysis of the positive and negative expression of Dll4 protein.

number of clinical researchers have found that CD44 plays an important role in the occurrence and development of tumors, and lately CD44V6 has attracted more attention due to its role in tumor invasion, metastasis, and prognosis. It has been reported that the overexpression of CD44V6 in various tumor tissues such as colorectal cancer, ovarian cancer, endometrial cancer, and other tumor tissues is closely related to tumor metastasis and poor prognosis $(8,9)$. In ovarian cancer, CD44V6 is not only related to the prognosis and recurrence of ovarian cancer but also the soluble CD44V6 in serum can be used as an effective indicator to evaluate prognosis and recurrence.

Some researchers have suggested that CD44V6 may also participate in tumor metastasis and invasion in osteosarcoma tissues (10), which is consistent with the results in this study. CD44V6 can bind with a variety of extracellular matrix components to mediate the adhesion of tumor cells to the extracellular matrix, which helps tumor cells to survive and develop in the primary lesion (11). In addition, CD44V6 also participates in a variety of transmembrane 
Table 4 Analysis of risk factors affecting prognosis and death of patients with osteosarcoma

\begin{tabular}{|c|c|c|c|c|c|c|}
\hline Variate & \multicolumn{3}{|c|}{ Single factor analysis } & \multicolumn{3}{|c|}{ Multiple-factor analysis } \\
\hline Gender: male (control: female) & 1.239 & $0.989-1.552$ & 0.724 & - & - & - \\
\hline Age: $\geq 25$ years (control $<25$ years) & 1.250 & $0.871-1.793$ & 0.697 & - & - & - \\
\hline Diameter of tumor: $\geq 5 \mathrm{~cm}$ (control $<5 \mathrm{~cm})$ & 1.260 & $0.850-1.868$ & 0.514 & - & - & - \\
\hline Enneking staging: stage II + III (control stage I) & 1.650 & $1.289-2.113$ & 0.023 & 1.749 & $1.261-2.426$ & 0.015 \\
\hline Metastasis: yes (control: no) & 1.672 & $1.273-2.196$ & 0.006 & 1.763 & $1.278-2.431$ & 0.004 \\
\hline DII4 positive (control: negative) & 1.692 & $1.269-2.257$ & 0.002 & 1.824 & $1.137-2.925$ & 0.012 \\
\hline
\end{tabular}

signal transmission processes, which affects intracellular cytoskeletal proteins and promotes neovascularization, adhesion to extracellular matrix, and invasion of tumors $(12,13)$. In this study, the results showed that the positive expression rate of CD44V6 in osteosarcoma tissue is higher than in normal bone tissue or osteochondroma, suggesting that the expression of CD44V6 is related to the occurrence, development, and malignant behavior of osteosarcoma. Consequently, the expression of CD44V6 might be used as an assistant index to assess the degree of tumor malignancy. In addition, results of this study also showed that the positive expression rate of CD44V6 of patients with tumor metastasis and Enneking stage II + III was significantly higher than those without metastasis, and at Enneking I. Furthermore, the survival rate of patients with a CD44V6 positive expression was significantly lower than those with a negative expression $(\mathrm{P}<0.05)$. Because CD44V6 is a key factor in the invasion and metastasis of osteosarcoma, its positive expression indicates a poor prognosis for patients, and a significantly reduced 2-year survival rate (14). It is suggested that CD44v6 is involved in the occurrence and metastasis of osteosarcoma, and can be used as a potential therapeutic target for malignant tumors.

The Dll4 protein contains 658 amino acid residues, eight EGF-like repeats, and Delta/Serrate/Lag-2 (DSL) domains outside the cell membrane (15). At present, it has been found that the blocking of DLL4-Notch1 signaling pathway can inhibit tumor growth, and the up-regulation of DLL4 expression in tumor tissues can be induced and regulated by hypoxia-inducible factors. At the same time, hypoxia can also induce the expression of the downstream genes of DLL4-
Notch1 signaling pathway, thus inducing angiogenesis (16). Pitulescu et al. have detected that the Dll4 protein is significantly overexpressed in liver cancer and bladder cancer, and this abnormal expression may be involved in tumor angiogenesis (17). Tumor angiogenesis is a key step in tumor growth and metastasis, because not only the growth of primary tumor depends on angiogenesis, but also the occurrence of metastasis and the progress of metastatic foci. The results in this study found that the positive expression rate of Dll4 in osteosarcoma tissue was significantly higher than that in normal bone tissue or osteochondroma $(\mathrm{P}<0.05)$, indicating that the occurrence of osteosarcoma is related to an abnormal increase in Dll4 expression. The expression of D114 was also found to be related to tumor differentiation, the Enneking stage, and metastasis $(\mathrm{P}<0.05)$, which further demonstrates that D1l4 plays an important role in the occurrence and development of osteosarcoma, and the tumor growth could be inhibited by blocking the activity of DLL4. In addition, the results of this study showed that the survival rate of patients with a Dll4 positive expression was also significantly lower than those with a negative expression $(\mathrm{P}<0.05)$, indicating that D114 might also be used as an effective indicator for evaluating the prognosis of patients. Many studies have shown that D1l4 and CD44V6 play important roles in the invasion and metastasis of a variety of malignant tumors. They are also one of several indicators that reflect the invasion and metastasis of tumor cells, and as such could be important indicators in evaluating the prognosis of patients with malignant tumors, especially osteosarcoma $(18,19)$. In the future treatment of osteosarcoma, we suggest that the expression levels of D114 and CD44V6 could be 
routinely tested so as to comprehensively assess the prognosis of the disease and provide reliable guidance for clinical treatment (20).

However, there are also some limitations in this study, such as a small sample size, a retrospective study, and inevitable deviation of the results. In addition, further prospective and large-sample studies on the research of DLL4 and CD44V6 in malignant tumors, clinical stages of osteosarcoma, and latest treatment methods need to be conducted.

In summary, Dll4 and CD44V6 show high positive expression rates in osteosarcoma, and these are closely related to the degree of tumor differentiation, Enneking stage, and metastasis. Furthermore, D114 and CD44V6 are risk factors that affect the prognosis and survival rate of patients. Therefore, in clinical settings, we suggest that the expression of Dll4 and CD44V6 could be tested to evaluate the prognosis of osteosarcoma patients.

\section{Acknowledgments}

Funding: This research was funded by Bethune: A special research fund for the treatment of pathological spinal fracture (BK-JP2020001), and the research project of the Affiliated Hospital of North Sichuan Medical College (2020JC029).

\section{Footnote}

Reporting Checklist: The authors have completed the REMARK reporting checklist. Available at http://dx.doi. org/10.21037/tcr-21-31

Data Sharing Statement: Available at http://dx.doi. org/10.21037/tcr-21-31

Conflicts of Interest: All authors have completed the ICMJE uniform disclosure form (available at http://dx.doi. org/10.21037/tcr-21-31). The authors have no conflicts of interest to declare.

Ethical Statement: The authors are accountable for all aspects of the work in ensuring that questions related to the accuracy or integrity of any part of the work are appropriately investigated and resolved. The study was conducted in accordance with the Declaration of Helsinki (as revised in 2013). The study was approved by institutional committee of Affiliated Hospital of North Sichuan Medical
College (2019ER(R)108-1) and informed consent was taken from all the patients.

Open Access Statement: This is an Open Access article distributed in accordance with the Creative Commons Attribution-NonCommercial-NoDerivs 4.0 International License (CC BY-NC-ND 4.0), which permits the noncommercial replication and distribution of the article with the strict proviso that no changes or edits are made and the original work is properly cited (including links to both the formal publication through the relevant DOI and the license). See: https://creativecommons.org/licenses/by-nc-nd/4.0/.

\section{References}

1. Anderson ME. Update on Survival in Osteosarcoma. Orthop Clin North Am 2016;47:283-92.

2. Zhuang M, Qiu X, Cheng D, et al. MicroRNA-524 promotes cell proliferation by down-regulating PTEN expression in osteosarcoma. Cancer Cell Int 2018;18:114-5.

3. Ma B, Li M, Zhang L, et al. Upregulation of long non-coding RNA TUG1 correlates with poor prognosis and disease status in osteosarcoma. Tumour Biol 2016;37:4445-55.

4. Spiegelberg D, Nilvebrant J. CD44v6-Targeted Imaging of Head and Neck Squamous Cell Carcinoma: AntibodyBased Approaches. Contrast Media Mol Imaging 2017;2017:2709547.

5. Chowdhury TA, Li K, Ramchandran R. Lessons learned from a lncRNA odyssey for two genes with vascular functions, DLL4 and TIE1. Vascul Pharmacol 2019;114:103-9.

6. Angulo P, Kaushik G, Subramaniam D, et al. Natural compounds targeting major cell signaling pathways: a novel paradigm for osteosarcoma therapy. J Hematol Oncol 2017;10:10-3.

7. Kushlinskii NE, Fridman MV, Braga EA. Molecular Mechanisms and microRNAs in Osteosarcoma Pathogenesis. Biochemistry (Mosc) 2016;81:315-28.

8. Farley JR, Kyeyune-Nyombi E, Tarbaux NM, et al. Alkaline phosphatase activity from human osteosarcoma cell line SaOS-2: an isoenzyme standard for quantifying skeletal alkaline phosphatase activity in serum. Clin Chem 1989;35:223-9.

9. Haylock A K, Nilvebrant J, Mortensen A, et al. Generation and evaluation of antibody agents for molecular imaging of CD44v6-expressing cancers. Oncotarget 2017;8:65152-70.

10. Deng Z, Niu G, Cai L, et al. The prognostic significance of CD44V6, CDH11, and -catenin expression in patients 
with osteosarcoma. Biomed Res Int 2013;2013:496193.

11. Sun J, Wang X, Fu C, et al. Long noncoding RNA FGFR3-AS1 promotes osteosarcoma growth through regulating its natural antisense transcript FGFR3. Mol Biol Rep 2016;43:427-36.

12. Uzan VR, Lengert Av, Boldrini É, et al. High Expression of HULC Is Associated with Poor Prognosis in Osteosarcoma Patients. PLos One 2016;11:e0156774.

13. Kamel WA, Sugihara E, Nobusue H, et al. SimvastatinInduced Apoptosis in Osteosarcoma Cells: A Key Role of RhoA-AMPK/p38 MAPK Signaling in Antitumor Activity. Mol Cancer Ther 2017;16:182-92.

14. Cheng HL, Hsieh MJ, Yang JS, et al. Nobiletin inhibits human osteosarcoma cells metastasis by blocking ERK and JNK-mediated MMPs expression. Oncotarget 2016;7:35208-23.

15. Djokovic D, Trindade A, Gigante J, et al. Incomplete D114/Notch signaling inhibition promotes functional angiogenesis supporting the growth of skin papillomas. BMC Cancer 2015;15:608.

Cite this article as: Xie H, Wang Y, Yu T, Jiang C, Wei P, Zhang Y. High expression of Dll4 and CD44V6 is associated with clinicopathological characteristics and poor prognosis in osteosarcoma patients. Transl Cancer Res 2021;10(2):10651072. doi: $10.21037 /$ tcr-21-31
16. Ubezio B, Blanco RA, Geudens I, et al. Synchronization of endothelial Dll4-Notch dynamics switch blood vessels from branching to expansion. Elife 2016;5:12167.

17. Pitulescu ME, Schmidt I, Giaimo BD, et al. Dll4 and Notch signaling couples sprouting angiogenesis and artery formation. Nat Cell Biol 2017;19:915-27.

18. Roth M, Barris DM, Piperdi S, et al. Targeting Glycoprotein NMB With Antibody-Drug Conjugate, Glembatumumab Vedotin, for the Treatment of Osteosarcoma. Pediatr Blood Cancer 2016;63:32-8.

19. Avnet $\mathrm{S}$, Lemma S, Cortini $\mathrm{M}$, et al. Altered $\mathrm{pH}$ gradient at the plasma membrane of osteosarcoma cells is a key mechanism of drug resistance. Oncotarget 2016;7:63408-23.

20. McKeage MJ, Kotasek D, Markman B, et al. Phase IB Trial of the Anti-Cancer Stem Cell DLL4-Binding Agent Demcizumab with Pemetrexed and Carboplatin as FirstLine Treatment of Metastatic Non-Squamous NSCLC. Target Oncol 2018;13:89-98.

(English Language Editor: D. Fitzgerald) 